Rabea Limbach*

\title{
Ost- und West-Fernsehen der 1950er- und 1960er-Jahre in der ARD Mediathek
}

https://doi.org/10.1515/bfp-2020-2044

Zusammenfassung: Im Herbst 2020 öffnet das Deutsche Rundfunkarchiv (DRA) online das Archiv des Deutschen Fernsehfunks (DFF), indem es historische Fernsehproduktionen des ehemaligen staatlichen Fernsehens der DDR zu einem gemeinschaftlichen Archivangebot aller Landesrundfunkanstalten der ARD beisteuert. Das neue Angebot in der ARD Mediathek startet zum Welttag des Audiovisuellen Kulturerbes am 27.10.2020 zunächst mit nicht-fiktionalen Fernsehproduktionen der 1950er- und 1960er-Jahre. Das Archivangebot soll in der Folge stetig ausgebaut werden, um einer breiten Öffentlichkeit einen digitalen $\mathrm{Zu}$ gang zu den öffentlich-rechtlichen Rundfunkarchiven zu ermöglichen.

Schlüsselwörter: Audiovisuelles Kulturerbe; DDR; Rundfunkarchiv

\section{East- and West German Broadcasting of the 1950s and 1960s in the ARD Media Library}

Abstract: As part of a joint online archive project of all public service broadcasters in the network of the ARD, the German Broadcasting Archive (Deutsches Rundfunkarchiv) will open the archive of the Deutscher Fernsehfunk (DFF) in autumn 2020. In this way historical television productions of the former state television of the GDR will be made digitally accessible. The new offering in the ARD media library will start on the World Day for Audiovisual Heritage on 27 October 2020, initially with nonfictional TV-productions of the 1950s and 1960s. In order to provide a broad public with access to the holdings of the broadcasting archives, it will be continuously expanded.

Keywords: Audiovisual heritage; GDR; broadcasting archive

\section{Einleitung}

Was sahen die Zuschauer in West- und Ostdeutschland in den 1950er- und 1960er-Jahren, wenn sie sich um den Fernsehapparat versammelten? Zum Welttag des Audiovisuellen Kulturerbes am 27. Oktober 2020 wird die deutsche Nachkriegszeit im Internet auf neue Weise erlebbar. Unter dem Branding „ARD Retro" ${ }^{\text {"A }}$ entsteht ein Archivangebot in der ARD Mediathek, das Zugang zu audiovisuellen Quellen aus den Archiven der Landesrundfunkanstalten ermöglicht. Das Deutsche Rundfunkarchiv, als älteste Gemeinschaftseinrichtung der ARD, steuert zu diesem Angebot Produktionen aus dem Archiv des Deutschen Fernsehfunks (DFF) bei, dem staatlichen Fernsehen der DDR. Damit wird es den Nutzern im Netz möglich, sowohl in Ost- als auch in West-Berichterstattung zu recherchieren. Ab Herbst 2020 werden in diesem Projekt zunächst nicht-fiktionale Fernsehbeiträge aus der Frühphase des deutschen Fernsehens dauerhaft zugänglich gemacht, darunter v.a. Nachrichtenformate und Magazinsendungen. ${ }^{2}$ Das Retro-Projekt der ARD bildet damit den systematischen Versuch, alle öffentlich-rechtlichen Rundfunkarchive in Deutschland für eine breite Öffentlichkeit digital zu öffnen.

\section{Aufbau eines ARD-Archivangebots in der ARD Mediathek}

Die öffentlich-rechtlichen Rundfunkarchive sind sich ihrer Rolle als Bewahrerinnen eines wichtigen Teils des audiovisuellen Kulturerbes Deutschlands bewusst. Dies haben die ARD-Anstalten und das ZDF bereits 2011 in ihrer „Freiwillige[n] Selbstverpflichtung [...] zur Umsetzung des Zusatzprotokolls ,Schutz von Fernsehproduktionen' zur ,Europäischen Konvention über den Schutz des audiovisuellen Kulturerbes" des Europarats" betont und sich dazu ver-

$1 \mathrm{https}$ //www.ardmediathek.de/ard/retro/.

2 Presseerklärung der ARD, unter: https://www.ard.de/home/die-ar d/presse/pressearchiv/ARD_Sender_oeffnen_ihre_Archive/6149808/ index.html.

*Kontaktperson: Dr. Rabea Limbach, rabea.limbach@dra.de 
pflichtet, dieses kulturelle Erbe nachhaltig zu bewahren. Nicht zuletzt vor dem Hintergrund der engen Zusammenarbeit der Rundfunkarchivare mit Fernseh-, Hörfunk- und Web-Redaktionen beobachten sie die sich wandelnden Bedürfnisse vieler Bevölkerungskreise in Bezug auf ihren Medienkonsum. Die Frage nach einer digitalen Öffnung der Rundfunkarchive ist daher unbestritten virulent - und wird seit vielen Jahren in der Öffentlichkeit, in Forschungsund Bildungseinrichtungen und in den Rundfunkanstalten diskutiert. In den vergangenen Jahren hat das Thema innerhalb der ARD nun erneut an Präsenz gewonnen. Dies spiegelt sich in den Tagungsprogrammen einiger Fachforen. Das Thema fand so zum Beispiel wiederholt Eingang in die Tagungsprogramme des Vereins für Mediendokumentation und -information (vfm), ${ }^{3}$ des Studienkreises Rundfunk und Geschichte ${ }^{4}$ oder in die Zugang-gestalten!Tagung des Jahres 2019.5

Die ARD-internen Überlegungen zur Öffnung der Archive wurden durch die letzte Änderung des Rundfunkstaatsvertrages im Jahr 2019 erleichtert, da die Bereitstellung von digitalen Archiven hierbei explizit in den Auftrag der Rundfunkanstalten integriert worden ist (§ 11d zu Telemedienangeboten). ${ }^{6}$ In der Umsetzung solcher Archivangebote ist im vergangenen Jahr der SWR mit dem Projekt SWR Retro vorangegangen. Auch dieses erste, systematische Archivöffnungsprojekt hat nachhaltig geholfen, das Thema noch stärker zu positionieren und daraus ein ARDweites Projektvorhaben zu entwickeln. ${ }^{7}$

\subsection{SWR Retro}

Mit SWR Retro ${ }^{8}$ ist es gelungen, einen größeren Fernseharchivbestand von mehr als sechs tausend historischen Beitragsvideos digital zugänglichzumachen. Die über die ARD Mediathek abrufbaren Videos werden dauerhaft vorgehalten. Das Angebot besteht aus Fernsehbeiträgen aus der Frühphase des Fernsehens im deutschen Südwesten -

3 Nachzulesen in den Tagungsberichten der Zeitschrift des Vereins info7.

$4 \mathrm{http}: / /$ rundfunkundgeschichte.de/bericht-translation-uebertragu ng-transmission/.

5 https://zugang-gestalten.org/dokumentation-2019/.

6 https://www.die-medienanstalten.de/fileadmin/user_upload/Rec htsgrundlagen/Gesetze_Staatsvertraege/Rundfunkstaatsvertrag_RSt V.pdf.

7 https://www.swr.de/unternehmen/kommunikation/pressemeldun gen/swrunternehmen-swr-retro-startet-2019-100.html.

8 https://www.ardmediathek.de/ard/sammlung/swr-retro-das-best e-aus-dem-archiv/6akPrCWyBP88kVkffgalJT/. vom Start des deutschen Fernsehens Mitte der 1950er-Jahre bis zum Ende des Jahres 1965. Darunter befindet sich vorrangig Nachrichtenberichterstattung aus den Sendereihen „Von Rhein, Main und Neckar“, „Abendschau“ und „Sport im Südwesten“, die von den Vorgängeranstalten des SWR - dem Süddeutschen Rundfunk (SDR) und dem Südwestfunk (SWF) - produziert und ausgestrahlt wurden. Hinzu kommen weitere, im Umfang überschaubarere Sendereihen, die stärker dem Bildungs-, Jugend- oder Unterhaltungsfernsehen zuzuordnen sind. Online gestellt wurden so zum Beispiel Folgen der Sendung „Was willst du werden?“, einer Sendung, die im Gemeinschaftsprogramm der ARD ausgestrahlt wurde und Jugendliche bei der Berufswahl unterstützen sollte. Stärker aus dem Bereich der unterhaltungsorientierten Kinder- und Jugendformate finden sich die Sendungen „Zirkus Dahl“ oder „Gefährlich Leben“, die von Arnim Dahl moderiert wurden. Dahl war ein international bekannter Stuntman, der seinen Ruhm vor allem waghalsigen Stunts in Kinofilmen und Werbekampagnen zu verdanken hatte. Hinzu kommen die ersten Folgen der Show „Die sechs Siebeng'scheiten“. In der Show traten jeweils zwei Schulklassen in einem Quiz gegeneinander an. Der Erfolg der Sendung führt dazu, dass sie in das ARD-Gemeinschaftsprogramm übernommen wurde und bis 1996 ein fester Teil des Fernsehprogramms des SWR blieb. Und schließlich finden sich im Angebot „SWR Retro“ auch einige Dokumentarfilme aus der Reihe „Zeichen der Zeit“. Mit ihnen macht die ARD Mediathek ausgewählte Filme der sogenannten „Stuttgarter Schule des Dokumentarfilms“ zugänglich, deren Filme zu ihrer Zeit über das Sendegebiet des SDR hinaus wiederholt ausgestrahlt wurden und regelmäßig politische Diskussionen bis in den Bundestag anstießen - da die Dokumentarfilmer in essayistischem Stil viele damals aktuellen Themen kritisch in den Blick nahmen.

Das Projekt SWR Retro konnte einen Weg aufzeigen, um die öffentlich-rechtlichen Rundfunkarchive in Deutschland zu öffnen. Hierfür hat sich im SWR ein abteilungsübergreifendes Team über mehrere Jahre mit vielfältigen Fragestellungen beschäftigt und die notwendigen Lösungskonzepte erarbeitet, die im Nachgang des Projektes auch innerhalb der ARD zur Diskussion gestellt worden sind. In den Erarbeitungsprozess waren neben Archivmitarbeitern und Online-Redakteuren Fachkollegen aus dem Justiziariat, dem Rechte- und Lizenzmanagement, der IT, der Programmplanung und der Kommunikationsabteilung eingebunden. Allein das vielfältige Know-how, das hier gebündelt wurde, verweist bereits auf die Komplexität dieser Vorhaben, in denen unter anderem vielfältige juristische Rahmenbedingungen, technische Systemlandschaften, Aufträge und Strategien der Rundfunkanstalten und 


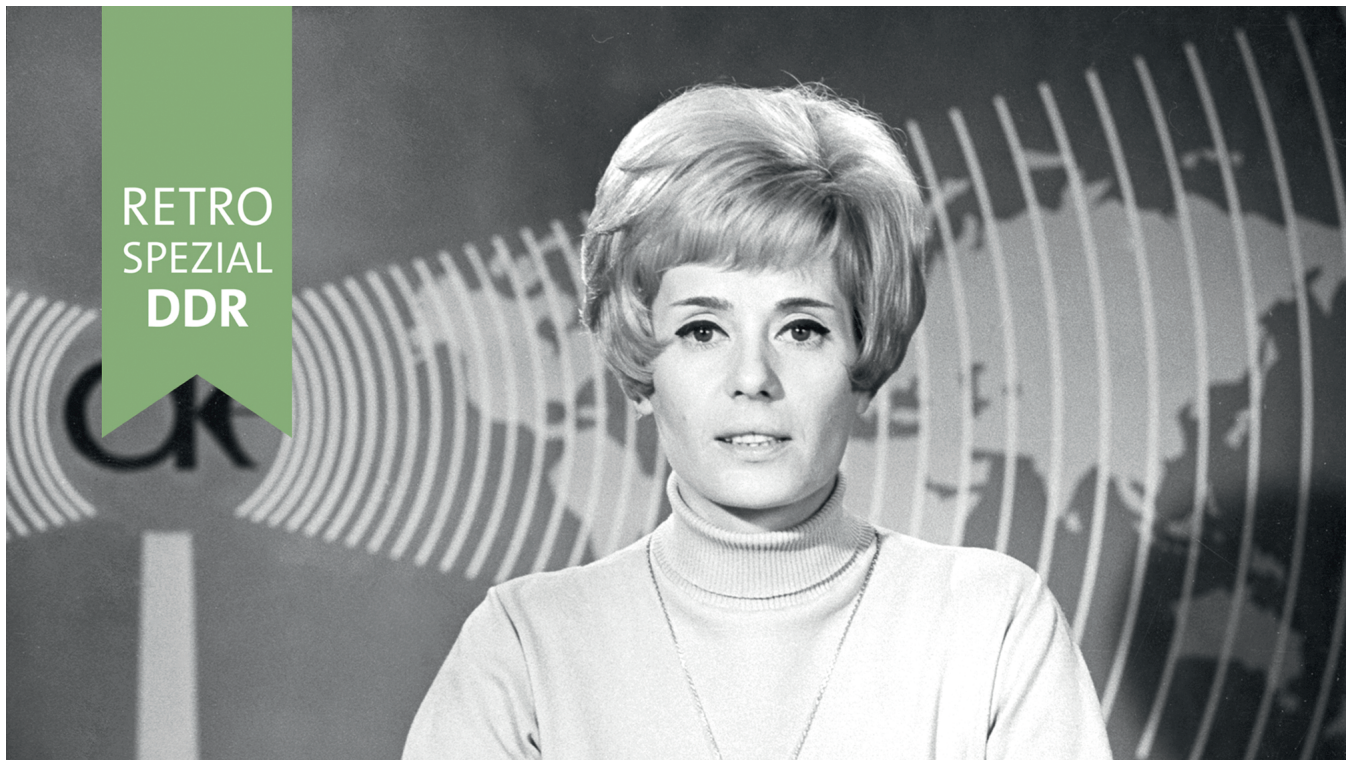

Abb. 1: Abbildung zum Mediatheksangebot DDR-Fernsehen. (c) ARD-Design/DRA/Waltraut Denger

nicht zuletzt die Überlieferungslage der Archivbestände in den Blick genommen werden müssen. ${ }^{9}$

\subsection{Gemeinsame Strategie zur digitalen Archivöffnung}

Vor dem Hintergrund von SWR Retro haben die ARDRundfunkarchive in Zusammenarbeit mit dem Deutschen Rundfunkarchiv, als einer von der ARD und Deutschlandradio getragenen Stiftung, im Winter 2019 beschlossen, sich eine gemeinsame Strategie für eine digitale Archivöffnung zu erarbeiten. Im Sommer 2020 haben die Intendanten der ARD nun über ein erstes, gemeinschaftliches Projektkonzept positiv entschieden, das vorsieht, dass alle ARD-Rundfunkanstalten ihre Fernseharchive über die ARD Mediathek - als zentraler Content-Plattform der ARD im Netz - öffnen. Das neue ARD-Archivangebot startet zum 27.10.2020 zum Weltgedenktag für das Audiovisuelle Kulturerbe (UNESCO) ${ }^{10}$ Im ersten Projektabschnitt werden nach dem Vorbild von SWR Retro Produktionen aus der Frühzeit des deutschen Fernsehens in die Mediathek überführt - darunter vor allem nichtfiktionale, aktualitätsbezogene und häufig regional ausgerichtete Sendereihen. Die parallele Zugänglichmachung ähnlicher Bestandsgruppen hat dabei den Vorteil,

9 Limbach (2018) $2 \mathrm{ff}$.

10 https://www.ard.de/home/die-ard/presse/pressearchiv/ARD_Se nder_oeffnen_ihre_Archive/6149808/index.html. dass die Nutzer zukünftig in die Lage versetzt werden, in der Mediathek vergleichend über große Teile der Fernsehberichterstattung aus allen Regionen Deutschlands zu recherchieren.

\section{DDR-Fernsehen in der ARD Mediathek}

Das DRA steuert zu dem neuen Archivangebot Beiträge aus dem Archiv des DFF bei, der staatlichen Fernsehanstalt der DDR. Das Archiv des DFF wurde nach der Auflösung der Fernsehanstalt im Zuge der Wiedervereinigung vonseiten der ARD der Stiftung Deutsches Rundfunkarchiv zur Bewahrung und Verwaltung übertragen. Seitdem ist das DRA beauftragt, die Bestände zu archivieren, zu erschließen, als Programmvermögen den Rundfunkanstalten zur Verfügung zu stellen und die Bestände auch für Wissenschaft und Forschung, Bildungsinstitutionen oder private Archivnutzer zugänglichzumachen.

Ausgerichtet an der gemeinsamen Strategie der ARDArchive steuert das DRA für das neue Mediatheksangebot zunächst frühe Fernsehproduktionen des DFF bei, der - parallel zur Entstehung der westdeutschen Fernsehprogramme - Mitte der 1950er-Jahre sein Programm aufnahm. Unter den bereitgestellten Archivbeständen befinden sich die Sendereihen „Aktuelle Kamera“ und „Prisma“ sowie einige frühe Sportformate. Die Aktuelle Kamera war die zentrale Nachrichtensendung des DDR-Fernsehens mit Berichten zu Ereignissen aus Politik, Wirtschaft und 
Kultur. ${ }^{11}$ Prisma, ein innenpolitisches Magazin, griff zweiwöchentlich aktuelle Probleme in der DDR auf. Das Magazin informierte ab 1963 über Fragen der gesellschaftlichen und wirtschaftlichen Entwicklung. ${ }^{12}$ Eine besondere, letztlich auch politische Rolle spielten die Sportsendungen in der DDR, nicht nur, weil sie zahlreiche Zuschauer fanden. Über weitere Ergänzungen des DFF-Angebotes in der ARD Mediathek wird innerhalb des DRA bereits beraten. ${ }^{13}$

Das Angebot zum DDR-Fernsehen in der Mediathek ermöglicht damit insbesondere Interessierten in den Bundesländern Brandenburg, Berlin, Mecklenburg-Vorpommern, Sachsen, Sachsen-Anhalt und Thüringen in der Geschichte ihres Lebensumfelds zu recherchieren - unter den Videos finden sich zum Beispiel Aufnahmen vom Abriss der Waisenbrücke in Berlin (Ost), der Wiedereröffnung der Dresdner Gemäldegalerie oder vom Bau der RappbodeTalsperre im Harz. Mit dem Angebot von Fernsehbeiträgen aus Ost und West fördert die ARD zudem die Auseinandersetzung mit der Geschichte der deutschen Teilung. In der Mediathek werden künftig Videos aus zwei unterschiedlichen Mediensystemen recherchierbar sein: dem öffentlichrechtlichen Rundfunksystem der Bundesrepublik und dem staatlichen Fernsehen der DDR, dessen Berichterstatter als „sozialistische Journalisten“ ideologisch geschult wurden und Partei für das im Aufbau befindliche „sozialistische Gesellschaftssystem “ der DDR und die Ziele der Partei- und Staatsführung ergreifen sollten. ${ }^{14}$

Die ARD Mediathek macht damit audiovisuelle Quellen zugänglich, die von dem abweichen, was dem Nutzer bisher in der ARD Mediathek angeboten werden: Es handelt sich nicht um Produktionen der Landesrundfunkanstalten der ARD oder ihrer Vorgängeranstalten. Vielmehr finden die Bestände in der Mediathek ihren Platz, da das Programmarchiv des DFF seit der Wende durch eine Archiveinrichtung der ARD, das Deutsche Rundfunkarchiv, archiviert wird. Die ARD übernimmt damit seit drei Jahrzehnten die Verantwortung, diesen Teil des audiovisuellen Kulturerbes Deutschlands zu bewahren und zugänglich zu machen. Daneben ist eine starke, rundfunkhistorische Verbindung zwischen der Überlieferung des DFF und der Überlieferung der ARD-Anstalten festzustellen. Seit den 1990er-Jahren greifen besonders die ARDRundfunkanstalten, deren Sendegebiete Gebiete der ehemaligen DDR umfassen (heute RBB, MDR, NDR), auf die Produktionen des DFF zurück, um ihr Programm zu gestalten. Viele DFF-Produktionen finden so als Ganzstückwie-

11 Bösenberg (2004).

12 Kreutz et. al. (1998) $206 \mathrm{ff}$.

13 https://www.dra.de/de/entdecken/fruehes-ddr-fernsehen/.

14 Bösenberg (2004) 11. derholungen im Fernsehprogramm neue Sendeplätze. Die Überlieferung des DFF dient den ARD-Rundfunkanstalten aber auch als Quellenpool, um sich z. B. in neu produzierten Dokumentationen mit der deutschen Geschichte seit dem Zweiten Weltkrieg auseinanderzusetzen. Sendungen aus dem DDR-Fernsehen werden zudem nicht nur wiederholt oder die überlieferten Filmmaterialen als Schnittmaterial weiterverwendet, vielmehr wurden und werden auch Sendereihen, die ehemals Teil des DFF-Programms waren, in den Fernsehprogrammen der ARD-Anstalten fortgesetzt. Hierzu zählen deutschlandweit ausgestrahlte Sendereihen wie das „Sandmännchen“ oder „Polizeiruf 110“, aber auch eine ganze Reihe von Sendungen, die in den regionalen Fernsehprogrammen der Landesrundfunkanstalten ausgestrahlt werden - wie z.B. „Umschau“ (MDR), „Visite“ (NDR) oder „Außenseiter-Spitzenreiter“ (MDR). Die Fernseh- und Hörfunkgeschichte der DDR und der Bundesrepublik sind hierdurch ebenso wie die Arbeit der ARD-Archive inkl. des DRA eng miteinander verzahnt. Eine Bereitstellung von DFF-Produktionen in der ARD Mediathek macht diese Zusammenhänge deutlich und ermöglicht es, einen übergreifenden Blick in die Fernseharchive und die deutsche Fernsehgeschichte zu werfen.

Nichtsdestotrotz darf nicht vergessen werden, dass die DDR-Fernsehbestände in der ARD Mediathek eine Besonderheit darstellen, da sie aus der staatlichen Fernsehanstalt eines nicht mehr existierenden, diktatorischen Staatssystems stammen. Innerhalb des ARD-Archivöffnungsprojektes haben wir uns vor diesem Hintergrund differenzierte Gedanken über die Ausspielung der Inhalte gemacht und zusammen mit Justiziariats- und Redakteurskollegen aus der ARD Konzepte zur Abgrenzung und Kontextualisierung der Bestände in der Mediathek erarbeitet. Dies war uns besonders wichtig, da das DRA zunächst Nachrichten und Magazinformate des DFF in die Mediathek einbringt, die vor dem Hintergrund der Geschichte der zwei deutschen Staaten und des Kalten Krieges unverkennbar ideologisch-propagandistisch geprägt sind und deren Berichterstattung von staatlicher Seite stark beeinflusst wurde. Um die Überlieferung des DFF von der Überlieferung der ARD-Anstalten abzugrenzen, haben wir daher in der Mediathek ein eigenes Angebot unter dem Namen „Retro Spezial: DDR“ entwickelt, in dem jedes Video farblich und grafisch eine klare Kennzeichnung erhält, damit bei der Recherche in der Mediathek auf den ersten Blick erkennbar ist, welche Videos der West- und welche der Ostberichterstattung zuzuordnen sind. 


\section{Exkurs: Rechtliche Hürden bei der Archivöffnung}

Möchte man ergründen, warum es so kompliziert ist, gemeinschaftlich finanzierte, öffentlich-rechtliche Rundfunkarchive in Deutschland digital für eine breite Öffentlichkeit zu öffnen, so wiegt hier sicherlich ein Aspekt am schwersten: die rechtlichen Rahmenbedingungen in Deutschland. Diese Rahmenbedingungen werde ich im Folgenden aus Sicht einer Historikerin, Dokumentarin und Rundfunkarchivmitarbeiterin kurz umreißen. Eine differenziertere Auseinandersetzung mit den juristischen Dimensionen möchte ich darüber hinaus meinen juristischen Fachkollegen im ARD-Archiv-Öffnungsprojekt überlassen. ${ }^{15}$

Bei der digitalen Öffnung von Rundfunkarchiven sind verschiedene rechtliche Dimensionen zu beachten, die zusammengenommen komplexe Rahmenbedingungen darstellen, die dazu führen, dass in hohem Maße Arbeit und Ressourcen in die Analyse dieser Rahmenbedingungen gesteckt werden muss - um überhaupt Handlungsspielräume für eine Archivöffnung zu finden. Hinzu kommt, dass es eine fast ebenso komplexe Herausforderung ist, die historischen Rechtskonstellationen im Fall von Fernseharchivbeständen und ihre Relevanz für eine Onlinestellung zu recherchieren, zu klären und bei Bedarf Rechte nachzuerwerben.

$\mathrm{Zu}$ den rechtlichen Dimensionen, die Einfluss auf Archivöffnungsprojekte der Rundfunkanstalten haben, zählen das Rundfunkrecht, das Wettbewerbsrecht, die Urheber- und Leistungsschutzrechte sowie die Persönlichkeitsschutz- und Datenschutzrechte.

Das Rundfunkrecht, zu denken ist hier an Rundfunkstaatsvertrag und ARD-Telemedienkonzepte, definiert den Auftrag der öffentlich-rechtlichen Rundfunkanstalten in Deutschland auch im Hinblick auf ihre Online-Angebote. ${ }^{16}$ In der Logik des Rundfunkrechts bilden die Webangebote der Rundfunkanstalten neben dem Fernseh- und Hörfunkprogrammen weitere Ausspielkanäle, für die geregelt wird, was die öffentlich-rechtlichen Medienanbieter dort tun sollen und dürfen. Und in diesem Kontext spielt auch das Wettbewerbsrecht eine Rolle, denn die Aufgabe öffentlichrechtlichen Rundfunkanstalten wird gerade deswegen definiert und abgegrenzt, um das Nebeneinander von öffentlich-rechtlichen und privaten Anbietern in Deutschland zu

15 Görgen (2019).

$16 \mathrm{Zu}$ den rechtlichen Grundlagen der Medienanstalten und Rundfunkstaatsvertrag: https://www.die-medienanstalten.de/service/rech tsgrundlagen. ermöglichen. Das Rundfunkrecht hat einen unmittelbaren Einfluss auf die Archivöffnungsprojekte, da es mit seinen Regelungen auf alle Online-Angebote der Rundfunkanstalten Anwendung findet. Dies liegt darin begründet, dass die Archive als Unternehmensarchive anzusehen sind, die dem Gesamtauftrag der Rundfunkanstalten untergeordnet werden - sie haben damit einen anderen Status als zum Beispiel öffentliche Archive, die einer eigenen Archivgesetzgebung unterliegen.

Auf das Rundfunkrecht in Deutschland ist so beispielsweise zurückzuführen, dass die öffentlich-rechtlichen Rundfunkanstalten zwar programmbegleitend vielfältige Inhalte in ihren Mediatheken ins Netz stellen dürfen, diese aber häufig nach kurzer Zeit wieder depublizieren müssen. Eine unbegrenzte Bereitstellung von Inhalten ist nach dem Rundfunkrecht nur sehr begrenzt möglich - und zwar nur für einen im Verhältnis zum Gesamtprogramm kleinen Anteil des Angebotes. Mit der Novelle des Rundfunkstaatsvertrages im Jahr 2019 sind die rundfunkrechtlichen Handlungsspielräume der Rundfunkanstalten im Hinblick auf die Öffnung ihrer Archive nun allerdings größer geworden, da der Aufbau von Online-Archiven offiziell als Teil des Auftrages der Rundfunkanstalten definiert wurde. Diesen neuen Auftrag gilt es nun wahrzunehmen und die Handlungsspielräume an dieser Stelle im Detail auszuloten, um der Öffentlichkeit in angemessener Weise Zugang zu der audiovisuellen Überlieferung zu ermöglichen.

Beschäftigt man sich mit der Überlieferung in den Rundfunkarchiven, so wird schnell deutlich, dass neben dem Rundfunkrecht auch Urheber- und Leistungsschutzrechte sowie Persönlichkeitsrechte bei einer Archivöffnung eine große Rolle spielen. In diesem Befund unterscheiden sie sich nicht von anderen Archiven, ${ }^{17}$ allein die Menge der Akteure und potenziellen Rechtsträger, die zu beachten sind, sind bei audiovisuellen Quellen ungleich höher als z. B. bei Schriftgutarchiven. Denn es liegt in der Natur der Sache, dass an Fernsehproduktionen eine Vielzahl von Menschen mitarbeitet. Diese stehen häufig in unterschiedlichen Vertragsverhältnissen zu den produzierenden Rundfunkanstalten (z.B. feste oder freie Mitarbeiter). Entsprechend sind die Werke mit den Einzelrechten vieler Menschen verbunden - man denke zum Beispiel an Autoren, Redakteure, Regisseure, Journalisten und Moderatoren, Schauspieler, Kameraleute, Cutter, Bühnenbildner, Ausstatter, Künstler oder Musiker. Bei den Fernsehproduktionen ist daher immer zu berücksichtigen, in welchem Vertragsverhältnis die Akteure zum Fernsehen

17 Klimpel (2020) $31 \mathrm{ff}$. 
standen und welche Rechte sie den Rundfunkanstalten in welchem Umfang eingeräumt haben - und das vor dem Hintergrund eines sich historisch wandelnden Urheberund Leistungsschutzrechtes, bei dem immer das Recht zu berücksichtigen ist, das zur Zeit der Entstehung der Produktionen Gültigkeit hatte. Und dabei ist festzustellen, dass es historisch relativ selten Vertragskonstellationen gab, die dazu führen, dass die Rundfunkanstalten eine Produktion bis heute auf allen Ausspielkanälen unbegrenzt nutzen dürfen. Vielmehr ist es häufig der Fall, dass die Rechte z.B. an verwendeten Musiken, eingeschnittenen Fremdmaterialien oder künstlerischen Darbietungen nur für eine begrenzte Weiternutzung eingeräumt wurden - zum Beispiel nur für eine feste Anzahl von Ausstrahlungen im Fernsehen. Und kommt man bei einzelnen Produktionen schließlich zu der Einschätzung, dass Rechte für eine geplante Nutzung der Produktion nacherworben werden müssten, beginnt bei historischen Fernsehformaten teilweise eine wahre Detektivarbeit, um herauszufinden, wer heute diese Rechte innehat - gerade wenn viele Urheber verzogen oder bereits verstorben sind. Wurden die Urheber schließlich ausfindig gemacht, so müssen mit ihnen individuell Vergütungen für die Nutzung ausgehandelt werden. Nur in einem Teil der Fälle können Rechte über pauschale Meldungen, z.B. bei Verwertungsgesellschaften, abgegolten werden.

Als weitere Herausforderung für den Aufbau großer Online-Archive kommt neben den Urheber- und Leistungsschutzrechten das Feld des Datenschutzes und der Persönlichkeitsrechte hinzu, das auch in anderen Archiven immer wieder zu Verunsicherung führt. ${ }^{18}$ Gerade die europäische Datenschutzgrundverordnung (DSVGO) hat hier bisher in unserer Wahrnehmung eher zu einer Zunahme der Unsicherheiten geführt, was im Netz zugänglich gemacht werden darf. In jedem Fall ist bei einer Archivöffnung immer zu analysieren, welche persönlichkeitsrechtlich relevanten Daten in Fernsehproduktionen oder den vorgehaltenen Metadaten zu den Quellen dokumentiert sind, um anschließend die Frage beantworten zu können, welche dieser Daten für eine Onlineveröffentlichung unbedenklich sind. Das Retro-Angebot in der Mediathek darf sich allerdings wie die übrigen Angebote der ARD auf das gesetzliche Medienprivileg stützen, das wiederum zu etwas anderen Handlungsspielräumen als bei anderen Archivangeboten im Netz führt.

Im Hinblick auf die DDR-Bestände haben wir uns im DRA intensiv mit dem Thema des Datenschutzes beschäftigt, da nicht nur viele Personen in der Fernsehbericht-

18 Nestl (2020) $7 \mathrm{ff}$. erstattung des DFF vorkommen und daran beteiligt waren, wir haben uns vielmehr auch die Frage gestellt, wie wir damit umgehen sollen, dass das Fernsehen der DDR eindeutig staatsnah war und durchaus parteilich über Personen berichtete. Dies konnte dazu führen, dass Menschen womöglich vor der Kamera anders agierten, als sie es in einem freiheitlich-demokratischen Gesellschaftssystem mit freien Medienanstalten getan hätten. Wir haben aus diesem Grund beschlossen, sensibel darauf zu achten, ob Menschen in der Berichterstattung offensichtlich diffamiert oder genötigt wurden, vor der Kamera zu sprechen zum Beispiel durch ein Filmen mit versteckter Kamera. Dies war uns besonders wichtig bei Menschen, die nicht aufgrund ihrer Prominenz im Fokus des öffentlichen Interesses von West- und Ostfernsehen standen, wie beispielsweise bei Politikern, bei denen es aus heutiger Sicht leichter einzuordnen ist, wenn über sie im Kontext des Kalten Krieges und des angespannten deutsch-deutschen Verhältnisses von Ost- oder Westfernsehen unausgewogen berichtet wurde.

Schließlich lässt sich im Hinblick auf das ARD-Archivöffnungsprojekt resümieren, dass die momentane Rechtslage in Deutschland es nur ermöglicht, Teile der Bestände der Rundfunkarchive einer breiten Öffentlichkeit im Netz zugänglich zu machen. Das Archivangebot der ARD bleibt damit vorläufig hinter der Idee zurück, möglichst breit die überlieferten Produktionen der Rundfunkanstalten zugänglich zu machen. Das Startangebot bildet ausgewählte Sendungen und Beiträge ab, bei denen es jetzt schon rechtlich möglich ist, sie im Netz zu veröffentlichen.

\section{Digitalisierung und Bereitstellung der Bestände im DRA}

Die Rundfunkarchive der ARD streben seit vielen Jahren eine umfassende Digitalisierung ihrer Bestände an - nicht zuletzt, da sie an die digitalen Produktionssysteme der Rundfunkanstalten angeschlossen sind. Um für die Redaktionen einen guten Service zu bieten, richten sie ihre Digitalisierungsstrategien darauf aus, alle angefragten Materialien zeitnah als File bereitstellen zu können - um in Situationen, in denen aufgrund aktueller Ereignisse eine schnelle Berichterstattung erforderlich wird, alles Notwendige zuliefern zu können. Zudem beschäftigen sich auch die Rundfunkarchive mit Fragen der langfristigen Sicherung ihrer Bestände, die teilweise auf Trägersystemen überliefert sind, die nicht zur langfristigen Archivierung geeignet sind (z.B. digitale Kassetten). Alle Rundfunkarchive verfügen vor dem Hintergrund seit Jahren laufen- 
der Massendigitalisierungsprojekte heute bereits über umfangreiche Filebestände mit historischen Produktionen in ihren Massenspeichern.

Wie die Landesrundfunkanstalten betreibt auch das DRA seit vielen Jahren die schrittweise Digitalisierung seiner Archivbestände - zuletzt im Rahmen einer großangelegten „Digitalisierungsstrategie 2020“ zur Massendigitalisierung von Hörfunk- und Fernsehproduktionen. Im Zentrum stehen dabei die Kernbestände des Archivs, wie die Bestände des DDR-Hörfunks und -Fernsehens sowie die Bestände zur Reichsrundfunkgesellschaft. Angeschlossen an die Digitalisierungsprojekte findet eine tiefergehende Erschließung der Produktionen statt. Das DRA ist vor diesem Hintergrund gut aufgestellt, um sich digital zu öffnen.

Allgemein ist in Bezug auf das Mediatheksprojekt mit Produktionen der 1950er- und 1960er-Jahre festzustellen, dass die Sendungen und Beiträge dieser Fernsehformate unterschiedlich gut überliefert sind. Dies ist zum einen auf die unterschiedlichen Archivierungspraktiken der Rundfunkarchive im Zeitverlauf zurückzuführen, da gerade in den neu gegründeten Fernsehanstalten der 1950er- und 1960er-Jahre nur selten Gesamtmitschnitte von Sendungen archiviert wurden. Fernsehen war gerade in seiner Frühphase meist Live-Fernsehen und wurde nur selten zur Programmdokumentation mitgeschnitten. In die Archive gelangten häufig nur vorproduzierte Beiträge, die in Sendungen eingespielt wurden und bei Bedarf weiterverwendet werden sollten. Hinzu kamen Materialengpässe bzw. hohe Materialkosten, die dazu führten, dass Trägermaterialien wie Tonbänder wiederverwendet und damit überspielt wurden. Die Chancen, dass eine Produktion bis heute vollständig überliefert ist, dürfte demnach bei aufwendig produzierten Spielfilmen, deren Wiederausstrahlungspotenzial die Fernsehanstalten früh erkannten, um ein vielfaches höher sein, als bei Nachrichtenberichten oder Liveübertragungen zum Beispiel von großen Sportereignissen oder Theaterstücken. ${ }^{19}$

Zu einer Besonderheit in der Überlieferung des frühen, deutschen Fernsehens führt das Phänomen, dass im Kontext des Kalten Krieges Teile des Fernsehprogramms des DDR-Fernsehens von westdeutscher Seite mitgeschnitten wurde - ebenso wie westdeutsches Fernsehen vonseiten der DDR einem Monitoring unterlag. ${ }^{20}$ So liegt heute im Deutschen Rundfunkarchiv ergänzend zur Überlieferung aus dem Archiv des Deutschen Fernsehfunks eine große Sammlung sogenannter Ostmitschnitte vor. Hierbei han-

19 https://www.dra.de/de/bestaende/ddr-rundfunk/fernsehen/. 20 Paulukat und Breitenborn (2007). delt es sich um Aufnahmen bei denen das Fernsehprogramm des DFF - mit Schwerpunkt auf aktuellen und politischen Sendungen - vom Sender Freies Berlin (SFB), dem Norddeutschen Rundfunk (NDR) und dem ZDF im Auftrag des Gesamtdeutschen Instituts von einem Fernsehgerät abgefilmt und archiviert wurde. Dieser Parallelüberlieferung verdanken wir heute, dass viel mehr Nachrichten- und Magazinbeiträge des Deutschen Fernsehfunks überliefert sind, als sie im unternehmenseigenen Archiv aufbewahrt wurden. Für die Sendereihe Aktuelle Kamera sind für die Jahre bis 1966 rund 30000 Einzelbeiträge im Deutschen Rundfunkarchiv überliefert. Für die Sendung Prisma, die erst im Jahr 1963 ins Programm aufgenommen wurde, rund 300 Beiträge. Die Überlieferung in Form von Ostmitschnitten führt zudem dazu, dass ein höherer Prozentsatz der Sendungsmoderationen mit überliefert ist, da diese ebenfalls live vor der Kamera eingesprochen und vom DFF selbst nur selten mitgeschnitten wurde. Die parallele Überlieferung hat allerdings auch einen Nachteil: Die Mitschnitte verfügen über eine mangelhaftere Video- und Audioqualität, da es sich nicht um die auf Film überlieferten Originale handelt.

\section{Ausblick: Perspektiven der weiteren Öffnung der Rundfunkarchive}

Vor zwei Jahren habe ich einen Artikel zur Öffnung der Rundfunkarchive in Deutschland veröffentlicht, der mit der etwas traurig anmutenden Bilanz endete, dass eine breite Öffnung der öffentlich-rechtlichen Rundfunkarchive unter den gegebenen Rahmenbedingungen kaum möglich sein wird, „kleine historische Film- und Hörfunksammlungen im Netz“ aber immerhin „ein Anfang“ wären. ${ }^{21} \mathrm{Ab}$ Herbst 2020 entsteht nun ein gemeinschaftliches OnlineArchivangebot der ARD mit Nachrichten und Magazinbeiträgen der 1950er- und 1960er-Jahre, das mittelfristig mehrere zehntausend Videos enthalten wird. Die Inhalte werden unbefristet vorgehalten. Das Embedding-Tool der Mediathek ermöglicht eine Einbettung der Videos auf Webseiten dritter. Permalinks ermöglichen, auf die Videos dauerhaft zuzugreifen und sie zum Beispiel auf Webseiten, in Präsentationen oder Publikationen zu verlinken und zuverlässig wieder abzurufen. Die Präsenz des ARD Mediatheksangebotes auf Smart-TVs verschiedener Anbieter ermöglicht das Abrufen der Archivvideos nicht nur über PCs

21 Limbach (2018) 8. 
und Smartphones, sondern auch über viele Fernsehgeräte. Diese Funktionalitäten schaffen eine breite Zugänglichkeit zu den Inhalten, die für Bildungsinstitutionen, Forscher und Kulturschaffende ebenso interessant sind, wie für geschichtsinteressierte Privatleute. Das Vorhaben, die ARDRundfunkarchive zu öffnen, ist damit erfreulich weit vorangekommen.

Die ARD möchte mit diesem Projekt nichtdestotrotz auch ein Zeichen setzen, um das Thema „Öffnung der öffentlich-rechtlichen Rundfunkarchive“ wieder stärker in der Öffentlichkeit zu positionieren. Entsprechend möchten wir parallel zu diesem Projekt auch auf die aus unserer Sicht unzureichenden, rechtlichen Rahmenbedingungen hinweisen, die es nach wie vor enorm erschweren, die Rundfunkarchive zu öffnen. Viele audiovisuelle Zeugnisse unserer Geschichte werden unter diesen Rahmenbedingungen weiterhin weitgehend ungenutzt in den Archiven liegen. Der Arbeitsgruppe ARD Archiv-Öffnung ist es jedoch ein nachhaltiges Anliegen, dem berechtigten Interesse der Öffentlichkeit, dass die Archive der durch unsere Gemeinschaft über einen Beitrag finanzierten Rundfunkanstalten geöffnet werden, nachzukommen. Es wäre daher wünschenswert, dass das gemeinsame Projektvorhaben der ARD-Rundfunkanstalten und des Deutschen Rundfunkarchivs zu einem breiteren Diskurs vor allem über das Urheberrecht in Deutschland führt und dabei nicht nur die Rechte einzelner Personen im Mittelpunkt stehen, sondern auch das Interesse unserer Gesellschaft an dem überlieferten, audiovisuellen Kulturerbe.

In der Fachliteratur rund um die Entwicklung des deutschen Urheberrechts werden bereits verschiedene Ansätze diskutiert, die helfen könnten, es Archiven in Deutschland zu erleichtern, sich digital zu öffnen. Hierunter finden sich auch einige aussichtsreichte Perspektiven für Rundfunkarchive mit audiovisueller Überlieferung. So wird zum Beispiel gerne der Vergleich mit dem amerikanischen Urheberrecht herangezogen, das über eine sogenannte Fairuse-Klausel verfügt, die es ermöglicht, urheberrechtlich geschützte Werke unter gewissen Bedingungen für nichtkommerzielle Zwecke zu nutzen, ohne Rechte nachzuerwerben..$^{22}$ Weitere Ansätze, die zu einer Erleichterung von Online-Archivprojekten führen könnten, bietet die europäische DSM-Richtlinie, ${ }^{23}$ die in Deutschland noch in nationales Recht umgesetzt werden muss. Denkbar für die Rechtsentwicklung in Deutschland wäre es demnach, dass ein System von Kollektivlizenzen ermöglichen könnte, für

22 Klimpel (2020).

23 Richtlinie (EU) 2019/790 des europäischen Parlaments und des Rates vom 17. April 2019 über das Urheberrecht und die verwandten Schutzrechte im digitalen Binnenmarkt. historische Rundfunkproduktionen vor einer Online-Stellung pauschale Lizenzgebühren an eine bestimmte Verwertungsgesellschaft zu zahlen. Die aufwendige Recherche nach einzelnen Rechtsträgern und der Erwerb von Einzelrechten würden damit entfallen. Ein anderer Ansatz zur Erleichterung der Öffnung von Rundfunkarchiven könnte in den Überlegungen zu ,vergriffenen Werken“ in der DSM-Richtlinie liegen: Auch Rundfunkproduktionen, die auf dem Markt nicht mehr erwerbbar und nirgendwo abrufbar sind, könnten als vergriffene Werke deklariert, in einem zentralen Register erfasst und über eine pauschale Gebühr und ohne Verfolgung eines kommerziellen Nutzen der Öffentlichkeit zugänglich gemacht werden. Die Urheber hätten dabei die Möglichkeit, gegen die Online-Veröffentlichung in einem begrenzten Zeitraum Einspruch zu erheben. Ein Beispiel für ein solches Verfahren in Deutschland bietet der Lizensierungsservice „Vergriffene Werke“ der Deutschen Nationalbibliothek für historische Monografien. ${ }^{24}$

Die ARD-Archivöffnung ist als langfristiges Projekt konzipiert: Neue Bestandssegmente in den Rundfunkarchiven, wie Hörfunkproduktionen oder Fernsehproduktionen späterer Entstehungszeiträume oder anderer Genres, werden in Zukunft im Fokus des Vorhabens stehen. Dabei befindet sich das Projekt stets auf der Suche nach Antworten auf die Frage, welche Produktionen mit vertretbarem Aufwand zugänglich gemacht werden können. Es kommt damit weiterhin viel konzeptionelle Arbeit auf uns zu. Wir hoffen, dass es uns damit in den kommenden Jahren gelingt, der Öffentlichkeit immer neue Bestandsgruppen aus den Rundfunkarchiven online zugänglich zu machen.

\section{Literaturverzeichnis}

Behmer, Markus; Bernard, Birgit; Hasselbring, Bettina (Hrsg.) (2014): Das Gedächtnis des Rundfunks. Die Archive der öffentlich-rechtlichen Sender und ihre Bedeutung für die Forschung. Wiesbaden: Springer.

Bösenberg, Jost-Arend (2004): Die Aktuelle Kamera (1952-1990). Lenkungsmechanismen im Fernsehen der DDR. Potsdam: Verlag für Berlin-Brandenburg. (Veröffentlichungen des Deutschen Rundfunkarchivs, 38).

Durantaye, Katharina de la; Raue, Benjamin (2020): Urheberrecht und Zugang in einer digitalen Welt - Urheberrechtliche Fragestellungen des Zugangs für Gedächtnisinstitutionen und die Digital Humanities. In: Recht und Zugang (RuZ). Zugang zum kulturellen Erbe und Wissenschaftskommunikation, 1, 83-94.

24 https://www.dnb.de/SharedDocs/Kontaktdaten/DE/vergriffene Werke.html. 
Durantaye, Katharine de la; Kuschel, Lina (2020): Vergriffene Werke größer gedacht: Art. 8-11 DSM-Richtlinie. In: ZUM (Zeitschrift für Urheber- und Medienrecht), 63 (8/9), 694-703.

Görgen, Markus (2019): Das Loch in der Wand. Öffnung der öffentlichrechtlichen Rundfunk-Archive im Lichte juristischer Machbarkeit. In: Info 7, (2), 21-23.

irights.info.de (2019): SWR-Archiv online: Audiovisuelle Dokumente für eine eindrückliche Beschäftigung mit der Geschichte. Interview mit Dr. Rabea Limbach. Verfügbar unter https://irights.inf o/artikel/swr-archiv-online-audiovisuelle-dokumente-fuer-eineeindrueckliche-beschaeftigung-mit-der-geschichte/29743.

Klimpel, Paul (2020): Recht und Geschichtsbild. In: Recht und Zugang (RuZ). Zugang zum kulturellen Erbe und Wissenschaftskommunikation, (1), 31-46.

Kramp, Leif (2015): Zur Situation der Rundfunkarchivierung in Deutschland. In: Rundfunk und Geschichte, (3-4), 11-24.

Kreutz, Anja; Löcher, Uta; Rosenstein, Doris (Hrsg.) (1998): Von „AHA“ bis „VISITE“. Ein Lexikon der Magazinreihen im DDR-Fernsehen (1952-1990/91). Potsdam: Verlag für Berlin-Brandenburg. (Veröffentlichungen des Deutschen Rundfunkarchivs, 13).

Kreutzer, Till (2016): Zugang für alle? Online-Rundfunkarchive auf rechtlicher Sicht. In: Info7, (3), 9-13.

Limbach, Rabea (2018): Archivbestände der Öffentlich-rechtlichen Rundfunkanstalten ins Netz! Ein Beitrag zu einem eigentlich unmöglichen Vorhaben? In: Info7, (3), 2-8.

Nestl, Andreas (2020): Zugang im Archiv. Möglichkeiten und Grenzen für ein offenes Archiv im digitalen Zeitalter. In: Recht und Zugang (RuZ). Zugang zum kulturellen Erbe und Wissenschaftskommunikation, (1), 5-15.

Paulukat, Susanne; Breitenborn, Uwe (2007): Signaturen des Kalten Krieges. Zur medienhistorischen und dokumentarischen Bedeutung der deutsch-deutschen Programmbeobachtungen. In: Rundfunk und Geschichte, (33), 29-37.
Rundfunkstaatsvertrag: Staatsvertrag für Rundfunk und Telemedien (Rundfunkstaatsvertrag - RStV) vom 31. August 1991 in der Fassung des Zweiundzwanzigsten Staatsvertrages zur Änderung rundfunkrechtlicher Staatsverträge (Zweiundzwanzigster Rundfunkänderungsstaatsvertrag) in Kraft seit 1. Mai 2019. Verfügbar unter https://www.die-medienanstalten.de/service/rechtsgrun dlagen.

Staats, Robert (2019): Kollektive Lizenzvergabe mit erweiterter Wirkung nach Art. 12 der DSM-Richtlinie - eine sinnvolle Lösung für Deutschland? In: ZUM (Zeitschrift für Urheber- und Medienrecht), 63 (8-9), 703-12.

Telemediengesetz. Verfügbar unter https://dejure.org/gesetze/TMG. Wagner, Hans-Ulrich (2019): Kulturelles Erbe. Das Online-Angebot „SWR Retro“ setzt einen Trend. In: epd medien, (49), 8-11.

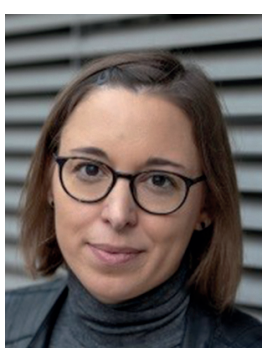

\section{Dr. Rabea Limbach}

Abteilung Information, Dokumentation \& Bestände

Deutsches Rundfunkarchiv

Marlene-Dietrich-Allee 20

D-14482 Potsdam

rabea.limbach@dra.de 